\title{
An Old Cytokine Against a New Virus?
}

\author{
Sandra Pellegrini ${ }^{1}$ and Gilles Uzé ${ }^{2}$
}

\section{To the Editor:}

$\mathrm{I}_{\mathrm{b}}^{\mathrm{N}}$ THE LAST 5 MONTHS, the COVID-19 pandemic has brought national health systems to critical emergency situations, and a very heavy price is being paid particularly by the elderly. The question of how to control and curb SARS-CoV-2 in the population is debated by governments worldwide with the help of scientific counselors, while researchers struggle to understand the many pathological effects in COVID-19. One after the other, countries face similar problems and implement unprecedented measures to limit the spread, but an extraordinary challenge is to identify infected patients, prevent the development of serious clinical symptoms, and treat those with severe respiratory distress. The effectiveness of the lockdown measures in the various countries will be difficult to evaluate and we may be confronted in a near future with a resurgence of the infection and appearance of new clusters. Likely a vaccine would solve most issues, but this will take several months. If, in the meantime, a treatment eliminated the risk of disease progression to severe pneumonia in the most sensitive population, COVID-19 would become an unpleasant but harmless infection. Here we compile a series of arguments strongly indicating that the complications of SARSCoV-2 infection are due to insufficient production of interferon (IFN) in the very early phase of the infection. Our considerations complement those recently put forward in Jamilloux and others 2020 and Sallard and others 2020 on the clinical evaluation of COVID-19 treatments. We concur on suggesting a systematic administration of exogenous IFN as early as possible after the molecular diagnosis of SARS-CoV2 in people who might be at high risk.

As for many viruses, the raison d'être of SARS-CoV-2 is to enter cells using specific cell membrane receptors, replicate its genetic material, and make proteins so as to produce numerous new virions that will infect more cells. However, to perform well the virus needs to counteract or evade an important innate barrier represented by IFN. No one would argue with the fact that, from fish to humans, type I IFNs are the most powerful natural arms (cytokines) to fight viruses. Evidence from studies in humans indicates that susceptibility to severe primary infections, notably flu, can be due to inborn defects in genes involved in the IFN-mediated antiviral response (Ciancanelli and others 2016).

Type I IFN is produced rapidly, in high amount and various flavors ( 1 IFN $\beta$ and many IFN $\alpha$ subtypes) by any type of cell infected by a virus or just triggered by viral components, such as viral RNA. Hence, within a few hours, IFN is synthesized in demand, is secreted in the extracellular milieu, and acts on the infected cells and all surrounding noninfected cells, making them resistant to the virus. This wave of high IFN production is transient and, in the best scenario, viral replication is severely hampered, and within a few days, with the help of the other actors of the immune response, the battle is over. However, if the cell mounts an inadequate response to the virus and/or if the virus hides or antagonizes IFN, the battle will continue. Infectious viruses will persist, and in the worst scenario an exaggerated production of immune mediators, cytokines, and chemokines and the recruitment of more immune cells will cause an excessive inflammatory response with local tissue damage. This scenario is well documented in murine models of infection with, for example, viruses such as flu, which possess strong and variegated weapons that reduce IFN production and oppose its antiviral activity at multiple levels (Haller and Weber 2007).

In the past 2 decades, extensive work on other coronaviruses, notably SARS-CoV and Middle East respiratory syndrome (MERS) isolates, has described the molecular mechanisms these viruses use to evade the IFN barrier, that is, IFN production and/or IFN response (Kopecky-Bromberg and others 2007; Perlman and Netland 2009). We will learn soon whether SARSCoV-2 uses similar or more sophisticated strategies to evade the IFN system in its human host. It is starting to dawn on us that, in in vitro experimental settings, $\mathrm{SARS}-\mathrm{CoV}-2 \mathrm{ORF} 6$ protein is one of the antagonists of IFN production (Yuen and others 2020).

Recent studies showed that SARS-CoV-2 and SARS-CoV replicate with the same kinetics in cells lacking the type I IFN genes cluster (monkey kidney epithelial Vero cells). Yet, when these cells were pretreated with exogenous IFN, SARS-CoV-2 was found to be much more sensitive to IFN than SARS-CoV (Blanco-Melo and others 2020; Lokugamage and others 2020). Among 22 antiviral agents tested, the most potent in reducing SARS-CoV-2 replication was IFN (Yuan and others 2020). This suggests that the new coronavirus may not be so talented in antagonizing environmental IFN, and that its rapid replication and high infectivity may relate, at least in part, to its ability to prevent the production of IFN by passively hiding from the sensing machinery of the host cell and/or by disrupting biochemical steps leading to IFN induction.

In line with that mentioned, SARS-CoV-2 was shown to be a poor inducer of IFN at least ex vivo on human lung

\footnotetext{
${ }^{1}$ Institut Pasteur, Department of Immunology, Cytokine Signaling Unit, Inserm U1221, Paris, France.

${ }^{2}$ CNRS UMR 5235, University of Montpellier, Montpellier, France.
} 
tissue (Chu and others 2020). Nevertheless, a recent report showed that IFN can be detected in the plasma of COVID19 patients (Hadjadj and others 2020). In a study of 50 patients distributed into 3 groups based on symptom severity, the authors reported that IFN $\alpha 2$ protein and IFN antiviral activity are highly significantly lower in the plasma of patients exhibiting critical symptoms than mild-tomoderate patients. Patients with intermediate (ie, severe but not critical) symptoms have intermediate IFN levels. Accordingly, the intensity of the transcriptional signature associated with IFN activity in circulating cells was found to inversely correlate with the severity of the clinical symptoms. Yet, when stimulated in vitro with IFN $\alpha 2$, blood cells from the different patient groups responded similarly to cells from healthy donors, suggesting that severe COVID-19 patients are affected in the capacity to produce IFN. This study included patients recruited at days 8-12 after the first clinical symptoms, a time when disease severity is most likely related to uncontrolled inflammation rather than to the absolute number of viruses in nasal swab or blood. Accordingly differences in the viral load in the 3 groups of patients were barely significant. Obviously, measurements at days 8-12 after the first symptoms do not inform on the level of IFN produced at the onset of infection. Hence, it can be assumed that the relatively low IFN measured in plasma of the less affected patients $(\sim 10 \mathrm{IU} / \mathrm{mL})$ is a residual quantity reflecting the extent of the initial peak of IFN produced when the virus was actively replicating. Thus the development of clinical symptoms would be related to the efficacy of the initial IFN production, that is, in severe cases, the production of IFN at the onset of infection might have been low. A recent study assessing plasma IFN $\alpha$ in few COVID-19 patients indeed suggests that the absence of detectable IFN $\alpha 2$ at days 8-10 postinfection is correlated with the need for invasive ventilation (Trouillet-Assant and others 2020). Additional arguments resulting from correlation analyses also support this hypothesis. When infected with SARS-CoV-2, the probability to develop severe symptoms is related to age and gender, and the ratio of male/female death rates tends to be higher in the older population. Age and gender are 2 biological variables known to be correlated with the production of type I IFN.

Aging is associated with considerable changes and a decline of immune function that leads to increased susceptibility to infections, particularly viral respiratory infections, in the elderly population. Often this decline is associated with lowgrade chronic inflammation. Impaired IFN secretion is likely to play a critical role in the increased susceptibility of the elderly to viral infections. A first in vitro study of human peripheral blood mononuclear cells (PBMCs) showed that the donor age influences the production of IFN $\alpha$ measured as antiviral activity (Abb and others 1984). As reviewed in Agrawal (2013), more recent studies demonstrated that, in human and mouse, the production of IFN by dendritic cells (DCs) and plasmacytoid dendritic cells (pDCs), which are stimulated with Toll-like receptor (TLR) agonists or infected with virus, is significantly impaired with age, but the ability to produce other inflammatory cytokines is not affected. Proposed mechanisms of impairment range from reduced number of cells, reduced expression of sensors, altered signaling, and transcriptional regulation.

Epidemiological data from the SARS-CoV 2002-2003 outbreak indicated gender-dependent differences in disease outcomes. Studies in the mouse paralleled those observed in patients and identified estrogen receptor signaling as critical for protection in females (Channappanavar and others 2017). In COVID-19 a similar gender-biased difference in morbidity and mortality is observed, whereas the proportion of males and females with confirmed infection is the same (Peckham and others 2020). Gender is an important biological variable in immune responses. Innate antiviral response and sensing of viral nucleic acids by some pathogenrecognition receptors, such as TLRs, differ between the genders (Klein and Flanagan 2016). A remarkable genderrelated difference in IFN $\alpha$ induction was observed in PBMC from healthy donors with a higher production in females, after stimulation of TLR7 but not TLR9. Since the TLR7 gene is on the $\mathrm{X}$ chromosome, it may escape $\mathrm{X}$ inactivation so that both alleles can be expressed simultaneously, resulting in higher levels in females than in males. This increased response is thought to contribute to the increased susceptibility of females to autoimmune diseases such as systemic lupus erythematosus. Estrogen-dependent modulation of TLR7 signaling has also been described (Griesbeck and others 2015). IRF5 is a transcription factor involved in the innate responses mediated by TLRs. pDCs from female humans and mice exhibit higher basal levels of IRF5 and produce more IFN $\alpha$ after TLR7 stimulation. Transcriptional regulation of IRF5 in female mice is under the control of estrogen. IRF5 gene polymorphisms are strongly correlated with the risk of systemic lupus erythematosus and elevated levels of IFN $\alpha$ in patients. These findings may contribute to the understanding of the higher prevalence of lupus in females.

The use of type I IFN as therapeutical drugs has been documented in SARS-CoV and MERS and initial trials are ongoing in COVID-19 (Jamilloux and others 2020; Sallard and others 2020). To date, a more complete clinical study is from Hong Kong (Hung and others 2020). This randomized phase 2 trial involved noncritically ill patients. One arm of the study enrolled patients $<7$ days after onset of symptoms, receiving either a combination regimen of lopinavir plus ritonavir, ribavirin and IFN $\beta$ (52 patients), or the combination but no IFN $\beta$ ( 24 patients, control). Significant differences in the primary endpoint (viral load, time to negative polymerase chain reaction from nasopharyngeal swab) and in the clinical score were found between the 2 groups, suggesting that an exogenous delivery of IFN can be efficient in controlling the early steps of the infection.

If we assume, as already discussed, that the development of critical clinical symptoms is due to a low endogenous IFN production, then an IFN given as a drug as early as possible after infection will be neutral to the large majority of patients who will not progress, but beneficial to the most fragile patients. The use of type I IFN in the clinic is not new. IFN has been administered alone or in combination for decades to treat chronic diseases, such as hepatitis B virus and hepatitis $\mathrm{C}$ virus infections, multiple sclerosis, and some cancers in patients of all ages. In these contexts, the adverse effects are well known and the severe effects are almost exclusively observed after long-term administration required by the chronic nature of the disease. In these diseases, we lack deep understanding of the beneficial and timely action of IFN, which may be directed to various target cells, including those of the immune system. In COVID-19, the issue of timing and target cell is straightforward, since the objective would be to induce a primary antiviral state. Hence a bolus injection at the early phase of infection may be sufficient. 
Interestingly, a recent study suggested that SARS-CoV-2 receptors (ACE2 and TMPRSS2) are upregulated by type I IFN (Ziegler and others 2020). If this observation is confirmed and, as intuitively suggested, if the binding of the virus to the cell surface is proportional to the quantity of its receptor, this could be a novel IFN-induced antiviral mechanism promoting the entry of virus into cells that are in an antiviral state. Intriguingly, ACE2 expression in many tissues was reported to be lower in the high-risk population (Chen and others 2020).

Presently, type III IFN is also considered as a possible antiviral therapy in COVID-19 (Prokunina-Olsson and others 2020). What distinguishes type I and type III IFNs is their receptors and the responding cells. Type I IFN receptors are ubiquitously expressed, whereas functional type III IFN receptors are restricted to epithelial lining at mucosal surfaces. Thus, administered type III IFN is expected to selectively target the respiratory tissues and have less side effects than type I IFN. However, as of today, we ignore whether the cells that are infected by SARS-CoV-2, that is, that express ACE2, have functional type III IFN receptors, and do respond to the cytokine. Besides, type III IFN may be more potent than type I IFN in disrupting lung epithelial repair during recovery from viral infection (Major and others 2020), and a sustained type III IFN signaling in inflamed mouse lung causes damage and susceptibility to secondary bacterial infection (Broggi and others 2020). Another issue is that we do not yet have the benefit of hindsight on the use of type III IFN in the clinic and have much less information concerning its pharmacokinetics and pharmacodynamics.

In conclusion, the production of type I IFN is an immediate innate response of the host to viral infections. High IFN rapidly counteracts virus replication and spread. An insufficient production of type I IFN at the onset of SARS-CoV-2 infection is likely to delay the kinetics of virus clearance. We propose that a type I IFN could be administered as early as possible after diagnosis as a single administration to patients for whom there is the slightest suspicion of complication.

\section{References}

Abb J, Abb H, Deinhardt F. 1984. Age-related decline of human interferon alpha and interferon gamma production. Blut 48(5):285-289.

Agrawal A. 2013. Mechanisms and implications of ageassociated impaired innate interferon secretion by dendritic cells: a mini-review. Gerontology 59(5):421-426.

Blanco-Melo D, Nilsson-Payant BE, Liu WC, Uhl S, Hoagland D, Moller R, Jordan TX, Oishi K, Panis M, Sachs D, Wang TT, Schwartz RE, Lim JK, Albrecht RA, tenOever BR. 2020. Imbalanced host response to SARS-CoV-2 drives development of COVID-19. Cell 181(5):1036.e9-1045.e9.

Broggi A, Ghosh S, Sposito B, Spreafico R, Balzarini F, Lo Cascio A, Clementi N, De Santis M, Mancini N, Granucci F, Zanoni I. 2020. Type III interferons disrupt the lung epithelial barrier upon viral recognition. Science; DOI: 10.1126/science.abc3545.

Channappanavar R, Fett C, Mack M, Ten Eyck PP, Meyerholz DK, Perlman S. 2017. Sex-based differences in susceptibility to severe acute respiratory syndrome coronavirus infection. J Immunol 198(10):4046-4053.

Chen J, Jiang Q, Xia X, Liu K, Yu Z, Tao W, Gong W, Han JJ. 2020. Individual variation of the SARS-CoV2 receptor ACE2 gene expression and regulation. Preprints, 2020030191, version 1. Available online at: https://www.preprints.org/ manuscript/202003.0191/v1 (accessed March 12, 2020).

Chu H, Chan JF, Wang Y, Yuen TT, Chai Y, Hou Y, Shuai H, Yang D, Hu B, Huang X, Zhang X, Cai JP, Zhou J, Yuan S, Kok KH, To KK, Chan IH, Zhang AJ, Sit KY, Au WK, Yuen KY. 2020. Comparative replication and immune activation profiles of SARS-CoV-2 and SARS-CoV in human lungs: an ex vivo study with implications for the pathogenesis of COVID-19. Clin Infect Dis. ciaa410; DOI: 10.1093/cid/ciaa410.

Ciancanelli MJ, Abel L, Zhang SY, Casanova JL. 2016. Host genetics of severe influenza: from mouse Mx1 to human IRF7. Curr Opin Immunol 38:109-120.

Griesbeck M, Ziegler S, Laffont S, Smith N, Chauveau L, Tomezsko P, Sharei A, Kourjian G, Porichis F, Hart M, Palmer CD, Sirignano M, Beisel C, Hildebrandt H, Cenac C, Villani AC, Diefenbach TJ, Le Gall S, Schwartz O, Herbeuval JP, Autran B, Guery JC, Chang JJ, Altfeld M. 2015. Sex differences in plasmacytoid dendritic cell levels of IRF5 drive higher IFN-alpha production in women. J Immunol 195(11):5327-5336.

Hadjadj J, Yatim N, Barnabei L, Corneau A, Boussier J, Pere H, Charbit B, Bondet V, Chenevier-Gobeaux C, Breillat P, et al. 2020. Impaired type I interferon activity and exacerbated inflammatory responses in severe COVID-19 patients. Preprint medRxiv; DOI: 10.1101/2020.04.19.20068015.

Haller O, Weber F. 2007. Pathogenic viruses: smart manipulators of the interferon system. Curr Top Microbiol Immunol 316:315-334.

Hung IF-N, Lung K-C, Tso EY-K, et al. 2020. Triple combination of interferon beta-1b, lopinavir-ritonavir, and ribavirin in the treatment of patients admitted to hospital with COVID19: an open-label, randomised, phase 2 trial. Lancet 395(10238): 1695-1704.

Jamilloux Y, Henry T, Belot A, Viel S, Fauter M, El Jammal T, Walzer T, François B, Sève P. 2020. Should we stimulate or suppress immune responses in COVID-19? Cytokine and anticytokine interventions. Autoimmun Rev; DOI: 10.1016/j.autrev.2020.102567.

Klein SL, Flanagan KL. 2016. Sex differences in immune responses. Nat Rev Immunol 16(10):626-638.

Kopecky-Bromberg SA, Martinez-Sobrido L, Frieman M, Baric RA, Palese P. 2007. Severe acute respiratory syndrome coronavirus open reading frame (ORF) 3b, ORF 6, and nucleocapsid proteins function as interferon antagonists. J Virol 81(2):548-557.

Lokugamage K, Hage A, Schindewolf C, Rajsbaum R, Menachery VD. 2020. SARS-CoV-2 is sensitive to type I interferon pretreatment. Preprint at bioRxiv; DOI: 10.1101/2020.03.07 .982264.

Major J, Crotta S, Llorian M, McCabe TM, Gad HH, Hartmann R, Wack A. 2020. Type I and III interferons disrupt lung epithelial repair during recovery from viral infection. Science; DOI: 10.1126/science.abc2061.

Peckham H, de Gruijter NM, Raine C, Radziszewska A, Ciurtin C, Wedderburn LR, Rosser EC, Deakin CT, Webb K. 2020. Sex-bias in COVID-19: a meta-analysis and review of sex differences in disease and immunity. Res Square; DOI: 10.21203/rs.3.rs-23651/v2.

Perlman S, Netland J. 2009. Coronaviruses post-SARS: update on replication and pathogenesis. Nat Rev Microbiol 7(6): 439-450.

Prokunina-Olsson L, Alphonse N, Dickenson RE, Durbin JE, Glenn JS, Hartmann R, Kotenko SV, Lazear HM, O’Brien TR, Odendall C, Onabajo OO, Piontkivska H, Santer DM, Reich NC, Wack A, Zanoni I. 2020. COVID-19 and emerg- 
ing viral infections: the case for interferon lambda. J Exp Med 217(5):e20200653.

Sallard E, Lescure FX, Yazdanpanah Y, Mentre F, PeifferSmadja N. 2020. Type 1 interferons as a potential treatment against COVID-19. Antiviral Res 178:104791.

Trouillet-Assant S, Viel S, Gaymard A, Pons S, Richard JC, Perret M, Villard M, Brengel-Pesce K, Lina B, Mezidi M, Bitker L, Belot A, group CHS. 2020. Type I IFN immunoprofiling in COVID-19 patients. J Allergy Clin Immunol; 10.1016/j.jaci.2020.04.029.

Yuan S, Chan C-Y, Chik K-H, Tsang, J-L, Liang R, Cao J, Tang K, Cai J-P, Ye Z-W, Yin F, To K.-W, Chu H, Jin D-Y, Hung I-N, Yuen K-Y, Chan JW. 2020. Broad-spectrum host-based antivirals targeting the interferon and lipogenesis pathways as potential treatment options for the pandemic coronavirus disease 2019 (COVID-19). Viruses 12, 628.

Yuen C-K, Lam J-Y, Wong W-M, Mak L-F, Wang X, Chu H, Cai J-P, Jin D-Y, To KW, et al. 2020. SARS-CoV-2 nsp13, nsp14, nsp15 and orf6 function as potent interferon antagonists. Emerg Microbes Infect:1-29; DOI: 10.1080/22221 751.2020.1780953.

Ziegler CGK, Allon SJ, Nyquist SK, et al. 2020. SARS-CoV-2 receptor ACE2 is an interferon-stimulated gene in human airway epithelial cells and is detected in specific cell subsets across tissues. Cell 181(5):1016.e19-1035.e19.

Address correspondence to: Dr. Sandra Pellegrini Institut Pasteur Department of Immunology Cytokine Signaling Unit, Inserm U1221 Paris 75724 cedex 15 France E-mail: sandra.pellegrini@pasteur.fr

Received 11 June 2020/Accepted 15 June 2020 